Pacific Journal of Mathematics

AMITSUR COHOMOLOGY OF ALGEBRAIC NUMBER RINGS 


\title{
AMITSUR COHOMOLOGY OF ALGEBRAIC NUMBER RINGS
}

\author{
DAvid E. DoBbS
}

\begin{abstract}
A bound is given for the order of the Amitsur cohomology group $H^{i}(S / R, U)$ corresponding to an extension $R \subset S$ of rings of algebraic integers. The effect of inflation on the ChaseRosenberg exact sequence involving Amitsur cohomology and split Brauer groups is also studied.
\end{abstract}

It is well known [9] that global class field theory, together with some results of Auslander-Goldman [2], leads to the determination of the split Brauer group $B(S / R)$ corresponding to an extension $R \subset S$ of rings of algebraic integers. Although the Amitsur cohomology group $H^{2}(S / R, U)$ is related to $B(S / R)$ by the Chase-Rosenberg exact sequence [5], $H^{2}(S / R, U)$ has only been computed in case $R=Z$ and $S$ is quadratic (see [10] and [8]). In this note we prove $H^{i}(S / R, U)$ is finite for all $i$ (Corollary 2.2) and, as in [8], derive further information in case $i=2$ by applying inflation to the Chase-Rosenberg sequence.

Throughout the paper, rings and algebras are commutative with unit elements and algebra homomorphisms are unitary. We assume familiarity with the Amitsur cohomology, Brauer group and Pic functors (see [4], [2] and [3] respectively) and with spectral sequences.

2. Finiteness of chomology. The aim of this section is to establish a bound for the order of Amitsur cohomology groups in the unit functor $U$ for extensions of rings of algebraic integers.

Proposition 2.1. Let $R$ be a Dedekind domain with quotient field $K, S$ the integral closure of $R$ in an n-dimensional separable field extension $L$ of $K$, and $T$ the integral closure of $R$ in a normal closure $F$ of $L / K$. Assume $U(T)$ can be generated by $m$ elements. Then, for all $i \geqq 0$, the Amitsur cohomology group $H^{i}(S / R, U)$ is finite, with order at most $n^{(m(n !) i)}$.

Proof. We first observe that the cochain group $C^{i-1}(S / R, U)=$ $U\left(S \otimes_{R} \cdots \bigotimes_{R} S\right)=U\left(S^{i}\right)$ is finitely generated for all $i \geqq 1$. By a standard argument [12, Chapter $V$, Theorem 7], $S$ and $T$ are modulefinite faithful $R$-projectives such that $S \otimes_{R} K \cong L$; hence the $R$-rank of $S$ is $n$. Let $G=\operatorname{gal}(F / K)$. Flatness provides injective $R$-algebra homomorphisms $S^{i} \rightarrow T^{i} \rightarrow F^{i}$; composition with the canonical isomor- 
phism $F^{i} \rightarrow \prod_{G^{i-1}} F$ (defined in $\left[4\right.$, p. 18]) yields an injection $S^{i} \rightarrow \Pi T$ and, hence, a monomorphism of abelian groups $U\left(S^{i}\right) \rightarrow \Pi U(T)$. Since $\Pi U(T)$ is finitely generated, so is $U\left(S^{i}\right)$.

$H^{i}(S / R, U)$, being a quotient of a submodule of $U\left(S^{i+1}\right)$, is therefore finitely generated. However, $H^{i}(S / R, U)$ is annihilated by $n$, the $R$-rank of $S$ [1, Theorem 6], and is therefore finite.

As $|G|=[F: K] \leqq n !$, it follows that $\prod_{G^{i}} U(T)$ can be generated by $m(n !)^{i}$ elements. By the elementary theory of abelian groups, the same conclusion holds for $C^{i}(S / R, U)$ and, hence, for its quotient $H^{i}(S / R, U)$. Since $H^{i}(S / R, U)$ is $n$-torsion, the result is immediate.

COROLLARY 2.2. If $R \subset S$ is an extension of rings of algebraic integers then, for all $i, H^{i}(S / R, U)$ is finite.

Proof. If $T$ is as above, a weak form of Dirichlet's unit theorem implies $U(T)$ is finitely generated, and the proposition applies.

3. Kernel of inflation. Let $f: S \rightarrow T$ be an $R$-algebra homomorphism and $J$ an abelian group valued functor defined on a full subcategory of $R$-algebras containing all the tensor products $S^{n}$ and $T^{n}$. The homomorphisms $J(f \otimes \cdots \otimes f): J\left(S^{n}\right) \rightarrow J\left(T^{n}\right)$ induce a map of Amitsur complexes $C(S / R, J) \rightarrow C(T / R, J)$ which yields inflation homomorphisms inf: $H^{n}(S / R, J) \rightarrow H^{n}(T / R, J)$.

In this section, we study the kernel of inf for the case $n=2$ and $J=U$, the unit functor. The principal result, Remark 3.3, complements the torsion result in [8, Theorem 2.7], and may be used with Corollary 4.4 below to yield information about cohomology of rings of algebraic integers.

We begin by recalling the following result.

Proposition 3.1. (Chase-Rosenberg [5, Theorem 7.2]). Let $R \rightarrow S$ and $S \rightarrow T$ be R-algebra homomorphisms, and let $J$ be a functor from $R$-algebras to abelian groups. For each $q \geqq 0$, let $J_{T}^{q}$ be the functor given by $J_{T}^{q}(A)=H^{q}\left(A \bigotimes_{R} T / A, J\right)$. Then there exists a first quadrant spectral sequence $H^{p}\left(S / R, J_{T}^{q}\right) \Rightarrow H^{p+q}(T / R, J)$.

The most important application of the proposition is to the case of an $R$-based topology (Definition [7, p. 86]) for which $J$ is a sheaf and $\{R \rightarrow T\}$ a cover. Then the natural transformation $J \rightarrow J_{T}^{0}$ is an equivalence. In particular, the $E_{2}^{p, 0}$ term of the above spectral sequence 
is just $H^{p}(S / R, J)$.

COROLLARY 3.2. Let $R \rightarrow S$ and $S \rightarrow T$ be R-algebra homomorphisms such that $T$ is faithfully flat over $R$. Then there is an exact sequence $H^{0}\left(S / R, U_{T}^{1}\right) \rightarrow H^{2}(S / R, U) \stackrel{\inf }{\rightarrow} H^{2}(T / R, U)$.

Proof. A standard spectral sequence argument, applied to the proposition with $J=U$, provides an exact sequence

$$
H^{0}\left(S / R, U_{T}^{1}\right) \rightarrow H^{2}\left(S / R, U_{T}^{0}\right) \stackrel{\text { edge }}{\longrightarrow} H^{2}(T / R, U) \text {. }
$$

However, $U$ is a sheaf in the faithfully flat $R$-based topology [5, Proposition 3.9(a)], and so the preceding remark identifies $U_{T}^{0}$ with $U$. Then [11, Lemma 1.1 and Proposition 1.6] identifies the edge homomorphism with inf, to complete the proof.

REMARK 3.3. Let $R \subset S \subset T$ be a tower of rings such that $T$ is faithfully flat over $R$ and $T$ is a rank $n S$-projective (rank defined in [3, p. 141]). Then ker [inf: $\left.H^{2}(S / R, U) \rightarrow H^{2}(T / R, U)\right]$ is $n$-torsion.

For the proof, it suffices to show $U_{T}^{1}(S)$ is $n$-torsion. However, [5, Corollary 4.6] yields natural isomorphisms

$$
\operatorname{ker}\left[\operatorname{Pic}(S) \longrightarrow \operatorname{Pic}\left(S \otimes_{R} T\right] \cong H^{1}\left(S \bigotimes_{R} T / S, U\right)\right.
$$

and

$$
\operatorname{ker}[\operatorname{Pic}(S) \longrightarrow \operatorname{Pic}(T)] \cong H^{1}(T / S, U) .
$$

Hence, $U_{T}^{1}(S)$ embeds in $H^{1}(T / S, U)$ which is $n$-torsion [1, Theorem $6]$, to complete the proof.

4. Direct limit arguments. We begin by recalling the ChaseRosenberg exact sequence of low degree obtained from the direct limit of spectral sequences given by Proposition 3.1.

Proposition 4.1. (Chase-Rosenberg [5, Theorem 7.6]). Let $S$ be a module-finite faithful and projective $R$-algebra. Then there exists an exact sequence natural in $S$ :

$$
\begin{gathered}
0 \longrightarrow H^{1}(S / R, U) \longrightarrow \operatorname{Pic}(R) \longrightarrow H^{0}(S / R, \operatorname{Pic}) \longrightarrow H^{2}(S / R, U) \longrightarrow \\
B(S / R) \longrightarrow H^{1}(S / R, \mathrm{Pic}) \longrightarrow H^{3}(S / R, U) .
\end{gathered}
$$

The isomorphism of Amitsur cohomology with split Brauer group in the case of fields is contained in the next result, a slight improvement of [5, Corollary 7.7]. 
$R$-algebra with $\operatorname{Pic}\left(S^{2}\right)=0$, then the natural map $H^{2}(S / R, U) \rightarrow B(S / R)$ is an isomorphism.

Proof. It suffices to prove $\operatorname{Pic}(S)=0$. Since composition of a face map $S \rightarrow S^{2}$ with the contraction map $S^{2} \rightarrow S$ is the identity on $S$, applying the functor Pic shows that the identity map on $\operatorname{Pic}(S)$ factors through $\operatorname{Pic}\left(S^{2}\right)=0$.

REMARK 4.3. Let $R \subset S$ be rings of algebraic integers whose quotient fields form an $n$-dimensional extension, and let $h$ be the class number of $S$. If $(h, n)=1$, then the canonical map $H^{2}(S / R, U) \rightarrow B(S / R)$ is a monomorphism.

Proof. Since $S$ is Dedekind, $\operatorname{Pic}(S)$ is isomorphic to the ideal class group of $S\left[3\right.$, Exer. 21, p. 181], and so $H^{\circ}(S / R$, Pic $)$ is $h$ torsion. As noted in the proof of Proposition 2.1, $S$ is a rank $n R$ projective and $H^{2}(S / R, U)$ is $n$-torsion. An application of Proposition 4.1 completes the proof.

For the remainder of this section, let $R$ be a ring of algebraic integers with quotient field $K, F$ an algebraic closure of $K$, and $A$ the ring of all algebraic integers inside $F$. We proceed to study direct limits of groups of the form $H^{n}(N / R, U)$, where $N$ ranges over the inclusion-directed collection of algebraic number overrings of $R$ contained in $A$. By cofinality, this may be viewed as taking direct limits over the map-directed collection of module-finite $R$-faithfully flat domains [7, Ch. III, Remark 3.1(b)]. Interest in such direct limits is partially due to the Cech description of cohomological field dimensions [7, Ch. I, Theorem 3.13].

In the finite $R$-based topology [7, p. 105], the functor $U$ is represented by $R\left[X, X^{-1}\right]$ which is an algebra-finite commutative cocommutative Hopf algebra over $R$. Consequently, [7, Ch. II, Remark 2.3(b)] shows that the canonical map $\lim H^{n}(N / R, U) \rightarrow H^{n}(A / R, U)$ is an isomorphism for all $n \geqq 0$.

Corollary 4.4. Applying lim to the sequence of Proposition 4.1 yields an isomorphism $H^{1}(A / R, \vec{N} U) \stackrel{\cong}{\rightarrow} \operatorname{Pic}(R)$ and an exact sequence $0 \rightarrow H^{2}(A / R, U) \rightarrow B(R) \rightarrow \lim H^{1}(N / R, \mathrm{Pic}) \rightarrow H^{3}(A / R, U)$.

Proof. If $P$ is a (not necessarily commutative) Azumaya $R$-algebra, let $L$ be a finite dimensional subextension of $F / K$ such that the class of $P \otimes_{R} K \otimes_{K} L \cong P \otimes_{R} L$ is trivial in $B(L)$. Let $M$ be the integral closure of $R$ in $L$. Since $B(M) \rightarrow B(L)$ is a monomorphism [2, 
Theorem 7.2], it follows that the class of $P \otimes_{R} M$ is trivial in $B(M)$. Thus $B(R)=\lim B(N / R)$, and the above discussion shows that we need only prove $\lim H^{0}(N / R$, Pic $)=0$. This, in turn, follows from the fact that $\lim \overrightarrow{\operatorname{Pic}}(N)=0$, which is an easy consequence of finiteness of class number (cf. [6, Theorem 20.14]).

\section{REFERENCES}

1. S. A. Amitsur, Complexes of rings, Israel J. Math., 2 (1964), 143-154.

2. M. Auslander and O. Goldman, The Brauer group of a commutative ring, Trans. Amer. Math. Soc., 97 (1960). 367-409.

3. N. Bourbaki, Algèbre commutative, chapitres 1-2, Hermann, Paris, 1962 (Act. scient. et ind. 1290).

4. S. U. Chase, D. K. Harrison and A. Rosenberg, Galois theory and Galois cohomology of commutative rings, Memoirs Amer. Math. Soc., No. 52, 1965.

5. S. U. Chase and A. Rosenberg, Amitsur cohomology and the Brauer group, Memoirs Amer. Math. Soc., No. 52, 1965.

6. C. W. Curtis and I. Reiner, Representation theory of finite groups and associative algebras, Wiley, New York, 1962.

7. D. E. Dobbs, Cech cohomological dimensions for commutative rings, Springer-Verlag, Berlin, 1970.

8. D. E. Dobbs, On inflation and torsion of Amitsur cohomology, Can. J. Math., Submitted.

9. R. M. Fossum, The Noetherian different of projective orders, Thesis, Universito of Michigan, 1963.

10. R. A. Morris, On the Brauer group of $Z$, Pac. J. Math., to appear.

11. B. Pareigis and A. Rosenberg, Addendum to "Amitsur's complex for purely inseparable fields," Osaka J. Math., 1 (1964), 33-44.

12. O. Zariski and P. Samuel, Commutative algebra, vol. I, Van Nostrand, Princeton, 1958.

Received December 14, 1970.

Rutgers University, The State University of New Jersey 



\title{
PACIFIC JOURNAL OF MATHEMATICS
}

\author{
EDITORS
}

\author{
H. SAMELSON \\ Stanford University \\ Stanford, California 94305 \\ C. R. HOBBY \\ University of Washington \\ Seattle, Washington 98105
}

J. DugundJI

Department of Mathematics

University of Southern California

Los Angeles, California 90007

RICHARD ARENS

University of California

Los Angeles, California 90024

\section{ASSOCIATE EDITORS}
E. F. BeCKenBaCH
B. H. NeUMANN
F. WOLF
K. Yoshida

\section{SUPPORTING INSTITUTIONS}

\author{
UNIVERSITY OF BRITISH COLUMBIA \\ CALIFORNIA INSTITUTE OF TECHNOLOGY \\ UNIVERSITY OF CALIFORNIA \\ MONTANA STATE UNIVERSITY \\ UNIVERSITY OF NEVADA \\ NEW MEXICO STATE UNIVERSITY \\ OREGON STATE UNIVERSITY \\ UNIVERSITY OF OREGON \\ OSAKA UNIVERSITY
}

\author{
UNIVERSITY OF SOUTHERN CALIFORNIA \\ STANFORD UNIVERSITY \\ UNIVERSITY OF TOKYO \\ UNIVERSITY OF UTAH \\ WASHINGTON STATE UNIVERSITY \\ UNIVERSITY OF WASHINGTON \\ AMERICAN MATHEMATICAL SOCIETY \\ NAVAL WEAPONS CENTER
}

The Supporting Institutions listed above contribute to the cost of publication of this Journal, but they are not owners or publishers and have no responsibility for its content or policies.

Mathematical papers intended for publication in the Pacific Journal of Mathematics should be in typed form or offset-reproduced, (not dittoed), double spaced with large margins. Underline Greek letters in red, German in green, and script in blue. The first paragraph or two must be capable of being used separately as a synopsis of the entire paper. The editorial "we" must not be used in the synopsis, and items of the bibliography should not be cited there unless absolutely necessary, in which case they must be identified by author and Journal, rather than by item number. Manuscripts, in duplicate if possible, may be sent to any one of the four editors. Please classify according to the scheme of Math. Rev. Index to Vol. 39. All other communications to the editors should be addressed to the managing editor, Richard Arens, University of California, Los Angeles, California, 90024.

50 reprints are provided free for each article; additional copies may be obtained at cost in multiples of 50 .

The Pacific Journal of Mathematics is published monthly. Effective with Volume 16 the price per volume (3 numbers) is $\$ 8.00$; single issues, $\$ 3.00$. Special price for current issues to individual faculty members of supporting institutions and to individual members of the American Mathematical Society: $\$ 4.00$ per volume; single issues $\$ 1.50$. Back numbers are available.

Subscriptions, orders for back numbers, and changes of address should be sent to Pacific Journal of Mathematics, 103 Highland Boulevard, Berkeley, California, 94708.

PUBLISHED BY PACIFIC JOURNAL OF MATHEMATICS, A NON-PROFIT CORPORATION

Printed at Kokusai Bunken Insatsusha (International Academic Printing Co., Ltd.), 270, 3chome Totsuka-cho, Shinjuku-ku, Tokyo 160, Japan. 


\section{Pacific Journal of Mathematics}

\section{Vol. 39, No. $3 \quad$ July, 1971}

William O'Bannon Alltop, 5-designs in affine spaces ................... 547

B. G. Basmaji, Real-valued characters of metacyclic groups ................. 553

Miroslav Benda, On saturated reduced products....................... 557

J. T. Borrego, Haskell Cohen and Esmond Ernest Devun, Uniquely representable semigroups. II.......................................

George Lee Cain Jr. and Mohammed Zuhair Zaki Nashed, Fixed points and stability for a sum of two operators in locally convex spaces ....................

Donald Richard Chalice, Restrictions of Banach function spaces ...............

Eugene Frank Cornelius, Jr., A generalization of separable groups ..............

Joel L. Cunningham, Primes in products of rings ......................

Robert Alan Morris, On the Brauer group of $Z$.

593

603

615

David Earl Dobbs, Amitsur cohomology of algebraic number rings ...............

Charles F. Dunkl and Donald Edward Ramirez, Fourier-Stieltjes transforms and

weakly almost periodic functionals for compact groups ...................

Hicham Fakhoury, Structures uniformes faibles sur une classe de cônes et

d'ensembles convexes ......................................

Leslie R. Fletcher, A note on C $\theta \theta$-groups.

Humphrey Sek-Ching Fong and Louis Sucheston, On the ratio ergodic theorem for

semi-groups............................................

James Arthur Gerhard, Subdirectly irreducible idempotent semigroups ...........

Thomas Eric Hall, Orthodox semigroups.....................

Marcel Herzog, $C \theta \theta$-groups involving no Suzuki groups ..........

669

687

John Walter Hinrichsen, Concerning web-like continua ..........

691

Frank Norris Huggins, A generalization of a theorem of F. Riesz.

695

Carlos Johnson, Jr., On certain poset and semilattice homomorphisms

703

Alan Leslie Lambert, Strictly cyclic operator algebras ...........

717

Howard Wilson Lambert, Planar surfaces in knot manifolds . . .

727

Robert Allen McCoy, Groups of homeomorphisms of normed linear spaces ....... 735

T. S. Nanjundiah, Refinements of Wallis's estimate and their generalizations ...... 745

Roger David Nussbaum, A geometric approach to the fixed point index .......... 751

John Emanuel de Pillis, Convexity properties of a generalized numerical range .... 767

Donald C. Ramsey, Generating monomials for finite semigroups ....

783

William T. Reid, A disconjugacy criterion for higher order linear vector differential equations...

Roger Allen Wiegand, Modules over universal regular rings...

Kung-Wei Yang, Compact functors in categories of non-archimedean Banach

spaces.

R. Grant Woods, Correction to: "Co-absolutes of remainders of Stone-Čech compactifications".

Ronald Owen Fulp, Correction to: "Tensor and torsion products of

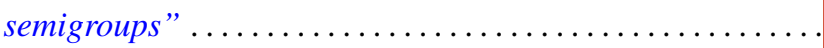

Bruce Alan Barnes, Correction to: "Banach algebras which are ideals in a banach 